\title{
POSITIVE DEFINITE MATRICES AND CATALAN NUMBERS, REVISITED
}

\author{
LOUIS W. SHAPIRO
}

\begin{abstract}
In this note a combinatorial correspondence is used to prove that the number of positive definite, tridiagonal, integral matrices of determinant 1 whose sub and super diagonals consist solely of ones is $C_{n}=\left(\begin{array}{c}2 n \\ n\end{array}\right) /(n+1)$. The correspondence is then further used to count such matrices by trace and also by number of ones on the main diagonal. Other related correspondences and results are given including those for determinant equal to 2,3,4 and 5 .
\end{abstract}

1. Introduction. In this paper we discuss $n \times n$ matrices which satisfy all the following properties. They are

(a) positive definite,

(b) tridiagonal,

(c) the sub and super diagonals consist only of ones,

(d) all entries are integers,

(e) the determinant is 1 .

We call such matrices suitable and the set of all such matrices $S_{n}$. In [7], Leighton and Newman prove the intriguing result that the number of suitable matrices is $C_{n}=\left(\begin{array}{c}2 n \\ n\end{array}\right) /(n+1)$, the $n$th Catalan number. They also include several interesting conjectures. The key to their proof is to go over to a set of integer sequences.

Define

$$
\left(a_{1}, a_{2}, \ldots, a_{n}\right)=A=\left[\begin{array}{cccccc}
a_{1} & 1 & 0 & 0 & \ldots & 0 \\
1 & a_{2} & 1 & & \ldots & 0 \\
0 & 1 & a_{3} & 1 & \ldots & 0 \\
& & & & \ldots & \vdots \\
\vdots & \vdots & \vdots & & a_{n-1} & 1 \\
0 & 0 & 0 & & 1 & a_{n}
\end{array}\right]
$$

and

$$
\left|a_{1}, a_{2}, \ldots, a_{n}\right|=|A|=\operatorname{det}\left(a_{1}, a_{2}, \ldots, a_{n}\right) .
$$

We start by summarizing some of the results in [7].

(A) For matrices satisfying (b) and (c),

$$
\left|a_{1}, a_{2}, \ldots, a_{k}\right|=a_{k}\left|a_{1}, a_{2}, \ldots, a_{k-1}\right|-\left|a_{1}, a_{2}, \ldots, a_{k-2}\right| \text {. }
$$

Received by the editors February 17, 1982 and, in revised form, February 9, 1983.

1980 Mathematics Subject Classification. Primary 05A15: Secondary 05B20. 
(B)

(4)

$$
|A|=\left|a_{1}, \ldots, a_{k}\right|\left|a_{k+1}, \ldots, a_{n}\right|-\left|a_{1}, \ldots, a_{k-1}\right|\left|a_{k}, \ldots, a_{n-k}\right| .
$$

For suitable matrices the following hold.

(C) If $A \in S_{n}$ then for some $k$,

$$
a_{k}=1
$$

(D)

$$
\begin{aligned}
\left(a_{1}, a_{2}, \ldots, a_{n}\right) & \in S_{n} \Leftrightarrow\left(a_{1}, a_{2}, \ldots, a_{k}+1,1, a_{k+1}+1, \ldots, a_{n}\right) \in S_{n+1} \\
& \Leftrightarrow\left(1, a_{1}+1, a_{2}, \ldots, a_{n}\right) \in S_{n+1} \\
& \Leftrightarrow\left(a_{1}, a_{2}, \ldots, a_{n-1}, a_{n}+1,1\right) \in S_{n+1} .
\end{aligned}
$$

The result (D) follows from:

(E) Let $A=\left(a_{1}, a_{2}, \ldots, a_{n}\right)$ satisfy (b) and (e) with $a_{k}=1$. Then $A$ is integrally congruent to [1] $\oplus\left(a_{1}, a_{2}, \ldots, a_{k-1}-1, a_{k+1}-1, \ldots, a_{n}\right)$.

Repeated application of (E) leads to:

(F) Every $A \in S_{n}$ is integrally congruent to the identity. We can now build up $S_{n}$ inductively by means of (D):

$$
\begin{gathered}
\left(S_{4}\right) \quad(1,2,3,1),(2,1,4,1),(1,4,1,2),(1,3,2,1),(4,1,2,2), \\
(3,2,1,3),(3,1,3,1),(2,3,1,2),(2,2,2,1),(1,2,2,2), \\
(2,1,3,2),(1,3,1,3),(3,1,2,3),(2,2,1,4) .
\end{gathered}
$$

For instance, $(2,2,1)$ becomes $(2,3,1,2)$ if we insert a 1 after the second 2. Now, inserting a 1 at the end of $(2,3,1,2)$ yields $(2, ., 1,3,1)$.

In $\S 2$ these results will be used to establish a one-to-one correspondence with triangulations of an $n$-gon. This leads to an interesting correspondence with binary trees.

In $\$ 3$ we prove the conjectures. In $\$ 4$ we discuss suitable matrices with the restriction on the determinant removed. This involves the matrix tree theorem and the counting of spanning trees.

2. The combinatorial correspondence. In $1758 / 9$ Euler proved, by a clever inductive argument, that the number of triangulations of a regular $(n+2)$-gon, by nonintersecting diagonals, is $C_{n}=\left(\begin{array}{c}2 n \\ n\end{array}\right) /(n+1)$. For $n=3$ we get the following graphs where we have distinguished the bottom edge and included the degrees of the other vertices:
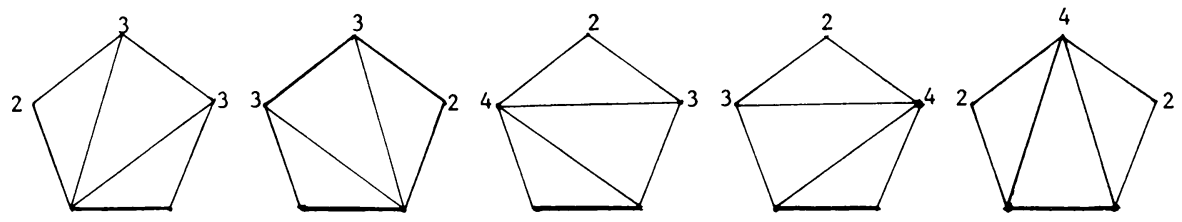
Listing the degree sequences we obtain

$$
(2,3,3) \quad(3,3,2) \quad(4,2,3)
$$

Now subtracting one from each entry we have

$$
(1,2,2) \quad(2,2,1) \quad(3,1,2) \quad(2,1,3) \quad(1,3,1)
$$

which is $S_{3}$ as in (9).

The basic idea is the same for all $n$. Take an $(n+2)$-gon, triangulate it, distinguish the bottom edge, write down the degree sequence (clockwise) of the other $n$ vertices, subtract one, and the result is an element of $S_{n}$. This indeed gives a bijection between triangulations of an $(n+2)$-gon and $S_{n}$.

We can show inductively that this works for all $n$ by noting two things. Recall (C) from [7], every $\left(a_{1}, a_{2}, \ldots, a_{n}\right) \in S_{n}$ has some $a_{k}=1$. Also every triangulation of an $(n+2)$-gon has at least two vertices of degree 2 and these two cannot be adjacent for $n>2$. Thus every triangulation has at least one vertex of degree 2 not incident with the distinguished edge.

Several items follow from this correspondence. Each vertex of degree 2 corresponds to an $a_{k}=1$. Also in [7], a break point is defined as the unique $k$ such that $\left(a_{1}, a_{2}, \ldots, a_{k-1}\right) \in S_{k-1}$ and $\left(a_{k+1}, \ldots, a_{n}\right) \in S_{n-k}$. This break point corresponds to the vertex opposite the distinguished edge in the triangle that includes this edge. For instance $*$ corresponds to the break point in:

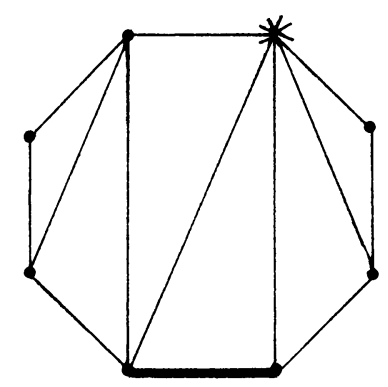

Having one of the standard Catalan settings we can easily find many, many more. See [4] for an extensive bibliography, and [3 and 10] for good exposition. We want an interpretation via binary trees and will use only an example to illustrate this correspondence:
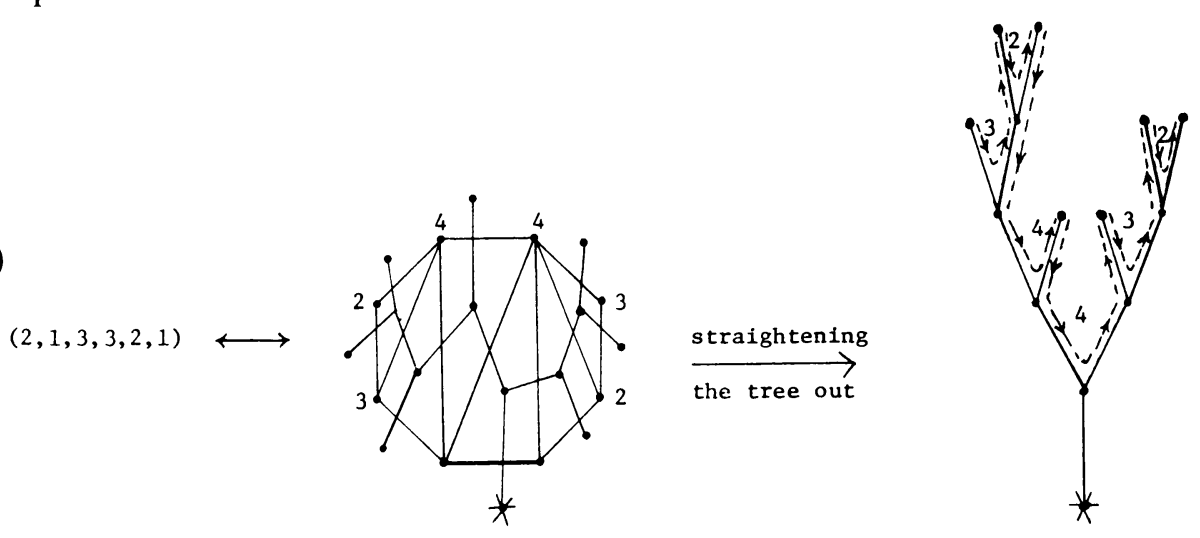
The degrees of the vertices represent the distance between successive end points (as a worm crawls around a tree [3]).

Another well-known setting for the Catalan numbers is parenthesizations of a product of length $n+1$. Again we will give one illustration:

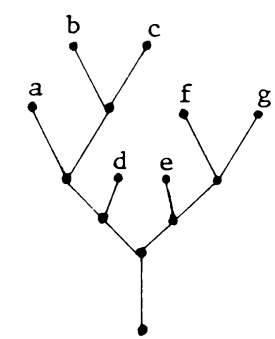

$$
\begin{gathered}
\rightarrow((a(b c)) d)(e(f g)) \\
1,0,2,2,1,0
\end{gathered}
$$

Count the number of parentheses, left or right, between $a_{i}$ and $a_{i+1}, i=1,2, \ldots, n$. Here we obtain $1,0,2,2,1,0$. Add 1 to each term, and an element of $S_{n}$ is obtained. Here $(2,1,3,3,2,1) \in S_{6}$.

Returning to suitable matrices there are several natural questions that occur.

(A) How many suitable matrices have trace $t$ ? The answer as conjectured in [7] is

$$
\frac{(3 n-t-1)(3 n-t-2)}{n-1}\left(\begin{array}{c}
t-n-1 \\
n-2
\end{array}\right) \text {. }
$$

(B) How many suitable matrices have $i$ ones along the main diagonal? Again the answer as conjectured in [7] is

$$
\frac{2^{n-2 i+1}(n-1) !}{(n-2 i+1) ! i !(i-1) !} .
$$

(C) How many suitable matrices have $a_{1}=a$ ? The answer is the ballot number

$$
B_{n, a}=\frac{a}{2 n+2-a}\left(\begin{array}{c}
2 n+2-a \\
n+1
\end{array}\right)
$$

The proofs of (A), (B) and (C) are given in the next section.

(D) The number of palindromic suitable matrices is

$$
\begin{cases}0 & \text { for } n \text { even } \\
\frac{2}{n+1}\left(\begin{array}{c}
n-1 \\
(n-1) / 2
\end{array}\right) & \text { for } n \text { odd }\end{cases}
$$

3. Some proofs. The proof of (B) follows quickly from results in the literature. The number of $a_{k}=1$ is the same as the number of terminal pairs $V^{2}$ in the corresponding binary tree. The number of such is

$$
\begin{aligned}
& \left(\begin{array}{c}
n-1 \\
2(i-1)
\end{array}\right) 2^{n-1-2(i-1)} C_{i-2} \\
& \quad=\frac{2^{n+1-2 i}(n-1) !}{(n+1-2 i) ! i !(i-1) !} .
\end{aligned}
$$


See Izbicki [5], Riordan [9] and Shapiro [10] for proofs of (19) or of the resulting identity (due to Touchard),

$$
\sum_{k=0}^{[n / 2]}\left(\begin{array}{c}
n \\
2 k
\end{array}\right) 2^{n-2 k} C_{k}=C_{n+1} .
$$

We start with the ballot numbers

\begin{tabular}{cc|cccccc}
$B_{l, k}$ & $l \backslash k$ & 1 & 2 & 3 & 4 & 5 & 6 \\
\cline { 2 - 7 } & 0 & 1 & 0 & 0 & 0 & 0 & 0 \\
& 1 & 1 & 1 & 0 & 0 & 0 & 0 \\
2 & 2 & 2 & 1 & 0 & 0 & 0 \\
& 3 & 5 & 5 & 3 & 1 & 0 & 0 \\
4 & 14 & 14 & 9 & 4 & 1 & 0 \\
& 5 & 42 & 42 & 28 & 14 & 5 & 1
\end{tabular}

(23) $B_{l, 0}=0=B_{l, l+m}, \quad m \geqslant 1, \quad B_{0,1}=1, \quad B_{l, k}=B_{l-1, k-1}+B_{l, k+1}$.

We need several facts about the ballot numbers arranged in this fashion. One is that the generating function for the $k$ th column is $x^{k-1}(C(x))^{k}$ where

$$
\begin{aligned}
C(x) & =1+x+2 x^{2}+5 x^{3}+\cdots \\
& =\sum_{k=0}^{\infty} \frac{1}{k+1}\left(\begin{array}{c}
2 k \\
k
\end{array}\right) x^{k}=\frac{1}{2 x}(1-\sqrt{1-4 x})
\end{aligned}
$$

is the generating function of the Catalan numbers. The second fact is that

$$
B_{l, k}=\frac{k}{2 l+2-k}\left(\begin{array}{c}
2 l+2-k \\
l+1
\end{array}\right) \text {. }
$$

See [6] for proofs.

For the proof of $(\mathrm{C})$ note that the condition $a_{1}=a$ corresponds to the following situation for $(n+2)$-gons:

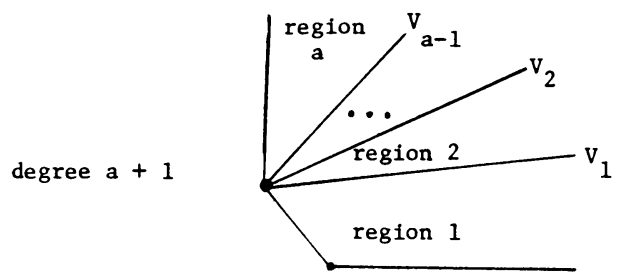

which corresponds to a collection of subpolygons:

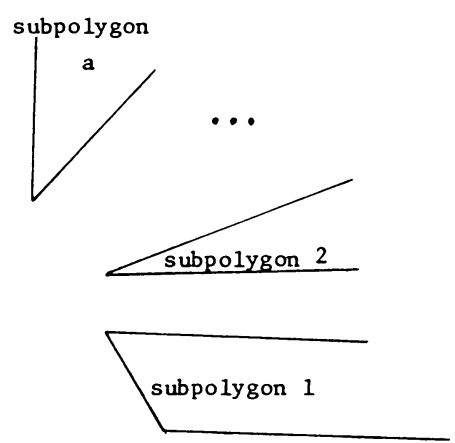


The generating function for the ways to triangulate a single polygon is $C(x)$. Thus for all $a$ polygons the generating function is $(C(x))^{a}$. The requirement that $v_{1}$, $v_{2}, \ldots, v_{a-1}$ exist gives the appropriate generating function for (26) as $x^{a-1} C(x)^{a}$. This gives the $a$ th column of the ballot numbers and thus

$$
B_{n, a}=\frac{a}{2 n+2-a}\left(\begin{array}{c}
2 n+2-a \\
n+1
\end{array}\right)
$$

is the number of possibilities for $n \times n$ suitable matrices with $a_{1}=a$.

The proof of the trace formula turns out to be similar in spirit. Note first that any triangulation by nonintersecting diagonals of an $(n+2)$-gon has total degree $4 n+2$. Let $d=d_{1}+d_{2}$ where $d_{1}$ and $d_{2}$ are the degrees of the two vertices of the distinguished edge. Then

$$
d+\sum_{i=1}^{n}\left(a_{i}+1\right)=4 n+2
$$

Thus

$$
t=\sum_{i=1}^{n} a_{i}=3 n+2-d .
$$

Break the polygon up into $d-4$ subpolygons as follows:

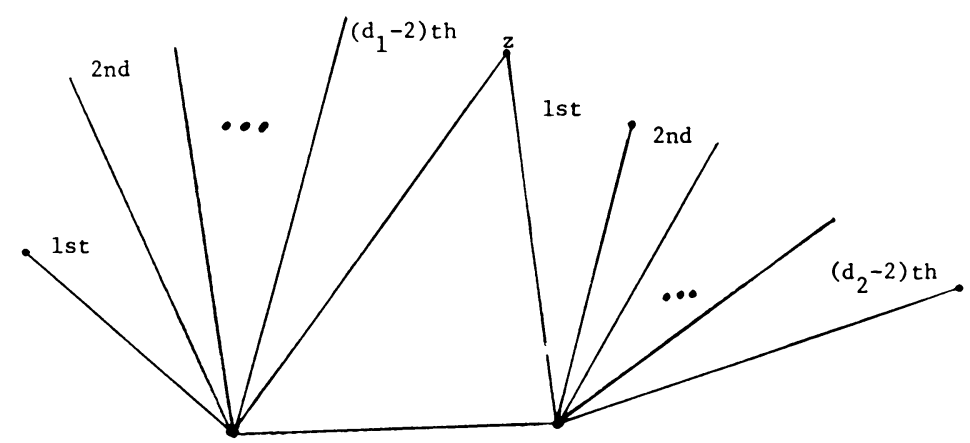

Define $d^{*}$ by $d^{*}=d-4$ and note that

$$
d^{*}=3 n-2-t
$$

while $2 n-3-d^{*}=t-n-1$. This arrangement has as its generating function $x^{d^{*}+1} C(x)^{d^{*}}$. Here the $d^{*}+1$ vertices that must be present are $v_{1}, v_{2}, \ldots, v_{d_{1-2}}$, $z, w_{1}, \ldots, w_{d_{2}-2}$.

The equation $d=d_{1}+d_{2}$ with $d_{1} \geqslant 2, d_{2} \geqslant 2$ has $d-3=d^{*}+1$ integral solutions. The appropriate generating function is

$$
(d-3) x^{d^{*}+1} C(x)^{d^{*}}=(d-3) x^{2} x^{d^{*}-1} C(x)^{d^{*}} \text {. }
$$

The coefficient of $x^{n}$ is

$$
\begin{aligned}
& (d-3) B\left(n-2, d^{*}\right) \\
& \quad=\left(d^{*}+1\right) \frac{d^{*}}{2(n-2)+2-d^{*}}\left(\begin{array}{c}
2(n-2)+2-d^{*} \\
(n-2)+1
\end{array}\right)
\end{aligned}
$$




$$
\begin{aligned}
& =\left(d^{*}+1\right) \frac{d^{*}}{2 n-2-d^{*}}\left(\begin{array}{c}
2 n-2-d^{*} \\
n-1
\end{array}\right) \\
& =\left(d^{*}+1\right) \frac{d^{*}}{2 n-2-d^{*}} \cdot \frac{2 n-2-d^{*}}{n-1} \cdot\left(\begin{array}{c}
2 n-3-d^{*} \\
n-2
\end{array}\right) \\
& =\frac{\left(d^{*}+1\right) d^{*}}{n-1}\left(\begin{array}{c}
2 n-3-d^{*} \\
n-2
\end{array}\right) \\
& =\frac{(3 n-1-t)(3 n-2-t)}{n-1}\left(\begin{array}{c}
t-n-1 \\
n-2
\end{array}\right) .
\end{aligned}
$$

This finishes the proof of (A).

The proof of (D) follows from similar reasoning. We omit the proof, mentioning only that the appropriate generating function is $x C\left(x^{2}\right)$.

4. Determinants greater than one. We now examine matrices which are suitable except that the determinant condition (e) is removed. The results are less complete but one interesting combinatorial construction comes in. We start with the illustration $(5,1,2,4,4)$. We start as before by removing ones:

$$
(5,1,2,4,4) \rightarrow(4,1,4,4) \rightarrow(3,3,4) .
$$

This has determinant 29. Note, however, that

(40) $\operatorname{det}\left(\begin{array}{ccccc}a_{1} & -1 & 0 & \cdots & 0 \\ -1 & a_{2} & -1 & \cdots & 0 \\ 0 & -1 & a_{3} & \cdots & 0 \\ 0 & 0 & -1 & \ddots & \end{array}\right)=\operatorname{det}\left(\begin{array}{ccccc}a_{1} & 1 & 0 & \cdots & 0 \\ 1 & a_{2} & 1 & \cdots & 0 \\ 0 & 1 & a_{3} & \cdots & 0 \\ \vdots & \vdots & \vdots & \\ 0 & 0 & 0 & \end{array}\right)$.

Now applying the matrix tree theorem [8] we find that the graph

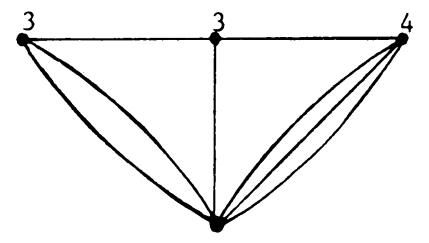

has 29 spanning trees.

In general, $\left(a_{1}, a_{2}, \ldots, a_{n}\right)$ with $a_{i} \geqslant 2$ is the number of spanning trees of the graph with vertex set $\left(v_{1}, v_{2}, \ldots, v_{n}, *\right)$ and with

(1) single edges between $v_{i}$ and $v_{i+1}, i=1,2, \ldots, n-1$,

(2) other edges, usually multiple, between $v_{i}$ and $*$ so that $\operatorname{deg}\left(v_{i}\right)=a_{i}$.

The general procedure follows the example. We start with $\left(a_{1}, a_{2}, \ldots, a_{n}\right)$ then first remove all ones until a $\left(b_{1}, b_{2}, \ldots, b_{m}\right)$ results, with $b_{i} \geqslant 2$ for all $i$. Then evaluate $\left|b_{1}, b_{2}, \ldots, b_{m}\right|$ or equivalently draw the equivalent graph and count spanning trees.

How many otherwise suitable matrices have determinant 3 ? 


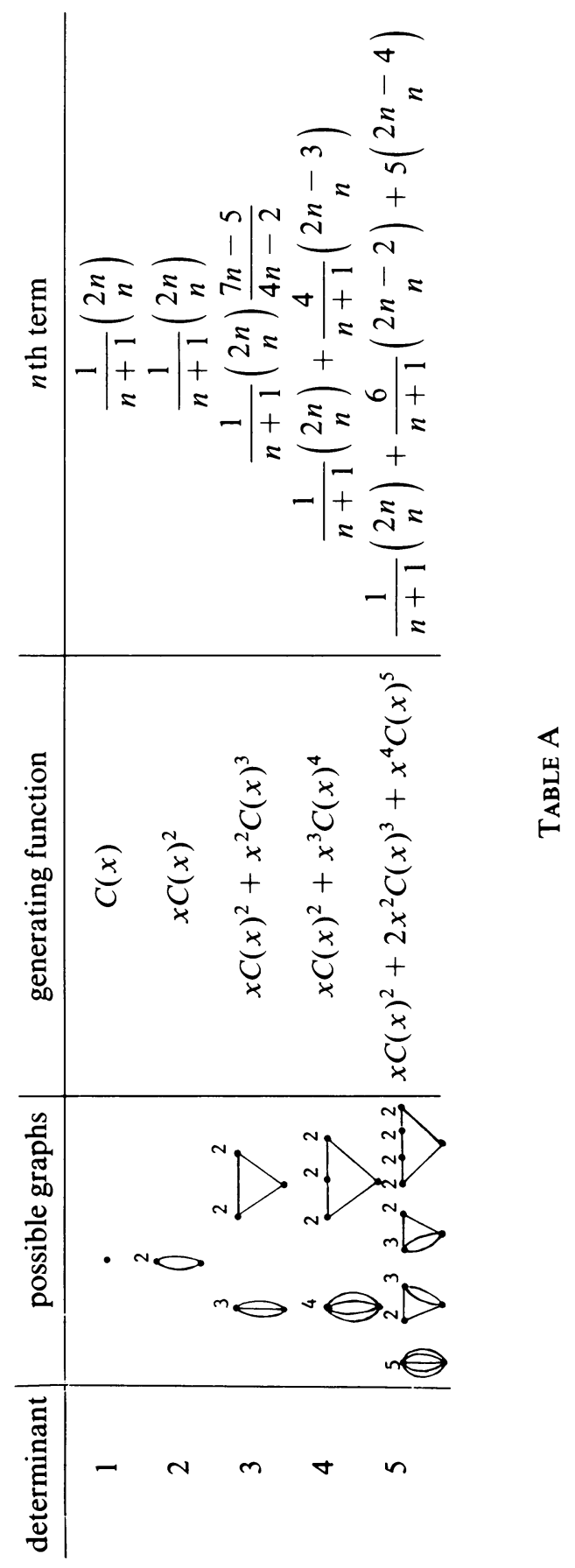


The following are the only possible such graphs with three spanning trees:
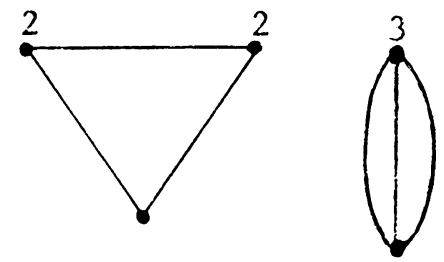

If we were now to add ones starting with the sequence $(2,2)$ we could add to the left, between the twos, or to the right. This gives $x^{2}(C(x))^{3}$ where the $x^{2}$ accounts for the start at $n=2$. The sequence (3) leads to the generating function $x C(x)^{2}$ and the total generating function is $x C(x)^{2}+x^{2} C(x)^{3}$.

This corresponds to adding the second and third columns of the $B_{l, k}$ table so the sequence starts $1,3,8,23,70, \ldots$, and the general term is

$$
\frac{2}{2 n+2-2}\left(\begin{array}{c}
2 n+2-2 \\
n+1
\end{array}\right)+\frac{3}{2 n+2-3}\left(\begin{array}{c}
2 n+2-3 \\
n+1
\end{array}\right)=\frac{1}{n+1}\left(\begin{array}{c}
2 n \\
n
\end{array}\right) \frac{7 n-5}{4 n-2} .
$$

A tabulation for small determinants goes as in Table A.

It is not clear how to find the number of possible graphs for arbitrary $n$. It is an interesting question, as is finding a unified setting for the spanning trees of this section and the binary trees of $\S 2$.

\section{REFERENCES}

1. R. Alter, Some remarks and results on Catalan numbers, Congressus Numer., vol. 3, Utilitas Math., Winnipeg, Man.,1975, pp. 109-132.

2. L. Euler, Opera Omnia 26 (1953), xvi-xviii.

3. M. Gardner, Catalan numbers: An integer sequence that materializes in unexpected places, Mathematical Games, Scientific American 234 (1976), 120-5, 132.

4. H. W. Gould, Research bibliography of two special number sequences, 1239 College Ave.. Morgantown, W. Va. 26505, 1976.

5. H. Izbicki, Uber Unterbaumes eines Baumes, Monatsh. Math. 74 (1970), 56-62.

6. D. Knuth, Fundamental algorithms, 2nd ed., vol. 1, Addison-Wesley, Reading. Mass., 1973, pp. $532-3$.

7. F. T. Leighton and M. Newman, Positive definite matrices and Catalan numbers, Proc. Amer. Math. Soc. 79 (1980), 177-181.

8. J. W. Moon, Counting labeled trees, Canad. Math. Mono., vol. 1, Canad. Math. Congress, 1970, pp. $39-46$.

9. J. Riordan, A note on Catalan parentheses, Amer. Math. Monthly 80 (1973), 904-906.

10. L. Shapiro, A short proof of an identity of Touchard's concerning Catalan numbers, J. Combin. Theory Ser. A 20 (1976), 375-6.

11. J. van Lint (ed.), Combinatorial theory seminar (Eindhoven Univ. of Technology, 1974), Lecture Notes in Math., vol. 382, Springer-Verlag, Berlin and New York, 1974, pp. 21-27.

Department of Mathematics, Howard University, Washington, D.C. 20059 